\title{
Microwave measurement system for dispersive dielectric properties of densely packed pellets
}

\author{
Livia Cerullo $^{\mathrm{a}}$, Johan Winges ${ }^{\mathrm{a}, *}$, Thomas Rylander ${ }^{\mathrm{a}}$, Tomas McKelvey ${ }^{\mathrm{a}}$, Lubomir Gradinarsky ${ }^{\mathrm{b}}$, Staffan Folestad ${ }^{\mathrm{b}}$, Mats Viberg ${ }^{\mathrm{a}}$ \\ ${ }^{a}$ Department of Signals and Systems, Chalmers University of Technology, SE-41296 Göteborg, Sweden \\ ${ }^{b}$ AstraZeneca RED Gothenburg, Pharmaceutical Technology and Development, SE-43183 Mölndal, Sweden
}

\begin{abstract}
We present a microwave measurement system that is intended for the estimation of the dispersive dielectric properties for densely packed pellets. In particular, we estimate the Debye parameters for the effective permittivity of a mixture of air and densely packed moist microcrystalline cellulose pellets for the frequency band $2.7 \mathrm{GHz}$ to $5.1 \mathrm{GHz}$. The Debye parameters are estimated by means of minimizing the misfit between the measured scattering parameters and the corresponding computed response given a model of the measurement system. This minimization is performed by means of a nonlinear iterative optimization procedure, which is supported by continuum sensitivities, and the optimum is found in 10-60 iterations for the cases presented in this article. We find that increasing moisture content yield an increase in the static permittivity and a decrease in the relaxation time.
\end{abstract}

Keywords: microwave measurement, dispersive media, permittivity, microcrystalline cellulose, gradient-based optimization, sensitivities, finite element method

\section{Introduction}

Fluidized beds are used in pharmaceutical industry for rapid drying, granulation, air-suspension coating among other things and the monitoring [1] of fluidized-bed processes is important in order to achieve high-quality pharmaceutical products at a low production-cost. In particular, the moisture content of the pellets $[2,3]$ in the bed influences the product quality and, consequently, it is important to be able to monitor and control the moisture content during the evolution of the fluidized-bed process. Another problematic aspect is the formation of so-called stagnation zones, where pellets do not fluidize and therefore are excluded from the circulation of material in the pharmaceutical process. Stagnation zones have a negative impact on the product quality and, consequently, it is desirable to monitor the bed and its distribution of pellets.

Near infrared (NIR) spectroscopy has been exploited for the measurement of moisture content, particle size and other quantities in pharmaceutical processes $[4,5,6]$. However, NIR spectroscopy performs the measurement on a rather small region of the process vessel and, therefore, it only provides local information on the process state. In addition, the NIR probe requires a free line of sight to the measurement region and, consequently, it is sensitive to material in the process that may attach to the probe and obstruct its field of view. Electric capacitance tomography (ECT) can be used to measure the effective permittivity in the bed region $[7,8,9,10]$. Although ECT provides information on the spatial variation of the effective permittivity, it

\footnotetext{
${ }^{*}$ Corresponding author

Email addresses: winges@chalmers.se (Johan Winges), rylander@chalmers.se (Thomas Rylander)
}

suffers from an intrinsically limited spatial resolution that is a consequence of the low frequency of operation [7]. An alternative technique that exploits stray fields of a microwave resonator is presented by Buschmüller et al. [11]. However, their method does not yield spatial resolution.

In this article, we present a first prototype of a microwave measurement system that is intended for the estimation of the effective permittivity of microcrystalline cellulose (MCC) pellets. The prototype can be modified as described by Cerullo et al. [12] in order to be used in a fluidized bed of Wurster type [13]. Our measurement equipment is non-intrusive in the sense that it does not need to be in physical contact with MCC pellets in order to perform the measurement and, in addition, it is possible to use the method in an on-line fashion for a fluidized-bed process. It can also provide information on spatial material distribution [12], which could potentially be used to identify stagnation zones. Our microwave measurement system features six rectangular waveguides that surround the measurement region and acts as antennas. The complete scattering matrix associated with the antenna ports is measured for a frequency band of nearly one octave. Given a numerical model of the measurement system, we minimize the misfit between the measured scattering parameters and the scattering parameters computed from our model, which depends on the parameters that describe the unknown dielectric medium. We also present a simple and accurate technique that makes it possible to reduce the residual between the measured response and the computational model of the system to a level of about $-30 \mathrm{~dB}$. Comparative tests for acrylic glass demonstrates that our proposed measurement technique yield estimated permittivities that are in good agreement with more conventional measurement procedures for cavity resonators. Finally, we demonstrate that it 
is feasible to estimate Debye parameters associated with MCC pellets and relate the values of the static permittivity and the relaxation time to the moisture content of the MCC.

\section{Measurement equipment}

Figure 1 shows the microwave measurement system, where Fig. 1(b) shows the equipment partially disassembled to display the interior of the measurement system. It consists of a metal cavity that features a measurement region at the center, which is formed by the intersection of six rectangular waveguides. The waveguides are of the type WR229 and we exploit the measurement system for the frequency band from $2.7 \mathrm{GHz}$ to $5.1 \mathrm{GHz}$. (This choice of frequency band yields a computationally manageable measurement problem with some possibilities to exploit the dispersive characteristics of moist materials used in pharmaceutical industry.) The rectangular waveguides are terminated by adapters connected to coaxial cables, where the coaxial cables are connected to a switch that allows for automatic measurement of the full $6 \times 6$ scattering matrix with the aid of a two-port network analyzer. This arrangement allows for the determination of the material distribution together with the material parameters and, in this article, we focus on the characterization of dispersive media.

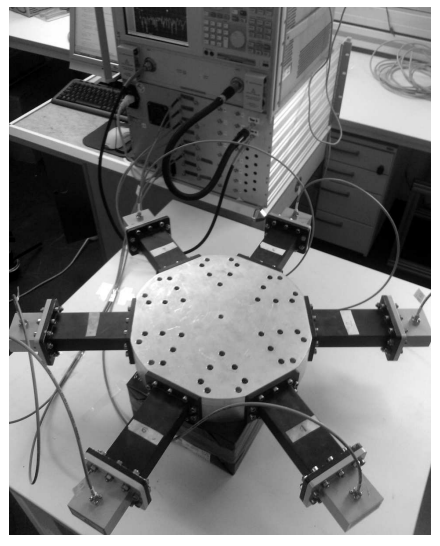

(a) Measurement system.

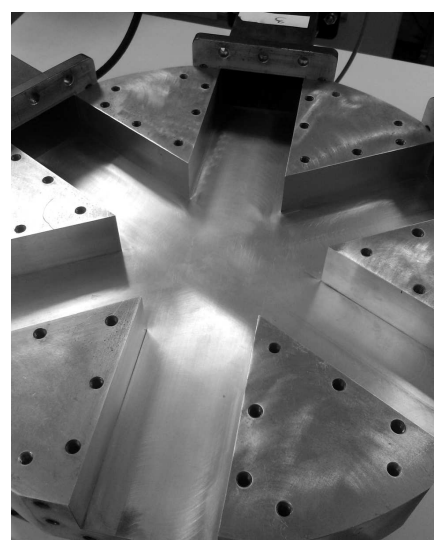

(b) Measurement region.
Figure 1: Microwave measurement system: (a) the assembled measurement system; and (b) the system when the lid is removed in order to display the measurement region at the intersection of six rectangular waveguides.

\subsection{Modeling in $2 D$}

Figure 2 shows the part of the measurement system that can be modeled accurately in two dimensions (2D) given that the dielectric samples subject to measurements also are 2D in the same manner, cf. the rectangular waveguides and their intersection shown in Fig. 1(b). Here, the computational domain $\Omega$ incorporates a portion of the rectangular waveguides together with the measurement region, which is enclosed by the dashed circle in Fig. 2.

Below, we give the main results for the modeling of the 2D problem in terms of the magnetic field $\boldsymbol{H}$, which is perpendicular to the $z$-axis. This problem can also be treated by means of

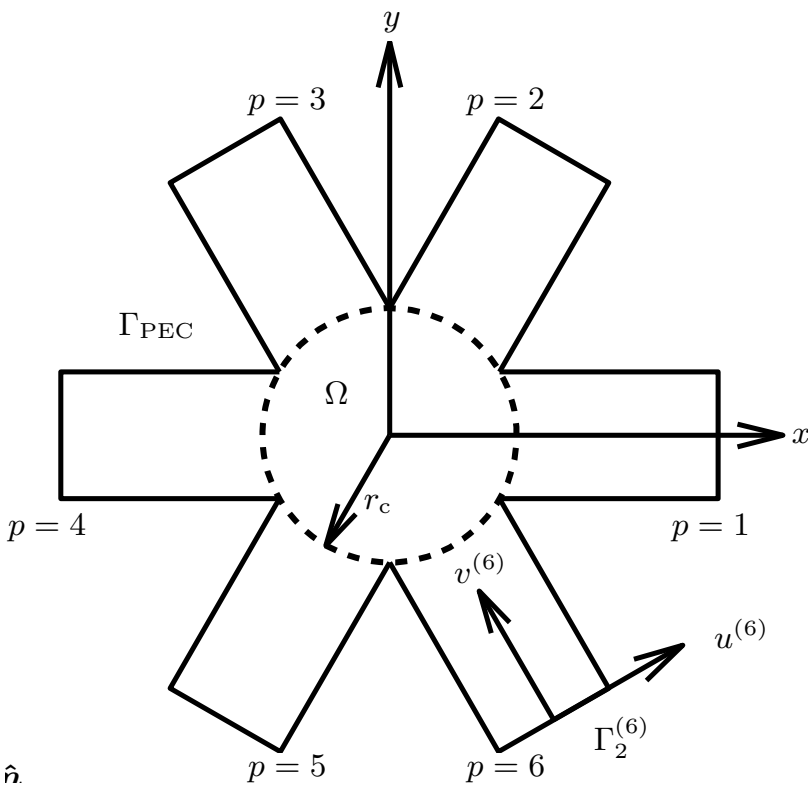

Figure 2: Geometry of the computational model of the microwave measurement system. Here, $\left(u^{(p)}, v^{(p)}\right)$ is the local coordinate system used for the Robin boundary condition at $\Gamma_{2}^{(p)}$ for port $p=1, \ldots, N_{\mathrm{p}}$. The dashed circle indicates the measurement region.

solving for the $z$-component of the electric field and, for further information on such formulations, the reader is referred to the book by Jin [14].

\subsubsection{Boundary value problem}

We seek the magnetic field $\boldsymbol{H}$ that satisfies the vector Helmholtz equation in the domain $\Omega$, i.e.

$$
\nabla \times\left(\epsilon_{\mathrm{c}}^{-1} \nabla \times \boldsymbol{H}\right)-\omega^{2} \mu_{0} \boldsymbol{H}=\mathbf{0} .
$$

Here, the magnetic field $\boldsymbol{H}$ is transverse to the cylinder axis, $\epsilon_{\mathrm{c}}$ is the complex permittivity subject to reconstruction, $\omega$ is the angular frequency and $\mu_{0}$ is the vacuum permeability.

We approximate the metal parts $\Gamma_{\mathrm{PEC}}$ of the boundary by means of a perfect electric conductor (PEC), which is modeled by the Neumann boundary condition

$$
\hat{\boldsymbol{n}} \times\left(\epsilon_{\mathrm{c}}^{-1} \nabla \times \boldsymbol{H}\right)=\mathbf{0} .
$$

The microwave system is operated in a frequency band that only allows for propagation of the fundamental $\mathrm{TE}_{10}$ mode in the rectangular waveguides, which are assumed to be of equal width $w$. The permittivity is $\epsilon_{0}$ in the waveguides and, in particular, close to the waveguide ports. Thus, inward and outward propagating waves can be modeled by a Robin boundary condition at the waveguide ports $\Gamma_{2}^{(p)}$ for $p=1,2, \ldots, N_{\mathrm{p}}$, where $N_{\mathrm{p}}=6$ in this article. At waveguide port $p$, we then have the boundary condition

$$
\hat{\boldsymbol{n}} \times\left(\epsilon_{\mathrm{c}}^{-1} \nabla \times \boldsymbol{H}\right)+\gamma \hat{\boldsymbol{n}} \times \hat{\boldsymbol{n}} \times \boldsymbol{H}=\boldsymbol{Q}^{(p)},
$$

where $\gamma=j \omega Z_{\mathrm{TE}_{10}}$ and $\boldsymbol{Q}^{(p)}=2 j \omega Z_{\mathrm{TE}_{10}} \hat{\boldsymbol{n}} \times \hat{\boldsymbol{n}} \times \boldsymbol{H}_{p}^{+}$. Here, $Z_{\mathrm{TE}_{10}}=\omega \mu_{0} / k_{\mathrm{v}}$ is the wave impedance and $k_{\mathrm{v}}^{2}=k_{0}^{2}-(\pi / w)^{2}$ is 
the wave number for the fundamental $\mathrm{TE}_{10}$ mode, where $k_{0}^{2}=$ $\omega^{2} \epsilon_{0} \mu_{0}$. Further, $\hat{\boldsymbol{n}}$ denotes the unit normal that points away from $\Omega$. We express the incident field $\boldsymbol{H}_{p}^{+}$at port $p$ in terms of the mode

$$
\boldsymbol{m}(u)=\hat{\boldsymbol{u}} \cos \left(\frac{\pi u}{w}\right)+\hat{\boldsymbol{v}} \frac{j \pi}{k_{\mathrm{v}} w} \sin \left(\frac{\pi u}{w}\right)
$$

such that $\boldsymbol{H}_{p}^{+}=H_{0 p}^{+} \boldsymbol{m}(u) \exp \left[-j k_{\mathrm{v}} v\right]$. The amplitude $H_{0 p}^{+}$for the incident magnetic field can be related to the corresponding electric field amplitude by $E_{0 p}^{+}=Z_{\mathrm{TE}_{10}} H_{0 p}^{+}$. Similar expressions apply to the magnetic field associated with the reflected wave, where suitable sign-changes also must be addressed.

\subsubsection{Numerical solution}

The field problem is solved by means of the finite element method (FEM) and it is formulated in terms of the weak form [14]: Seek $\boldsymbol{H} \in H(\operatorname{curl} ; \Omega)$ such that

$$
a(\boldsymbol{w}, \boldsymbol{H})=b(\boldsymbol{w})
$$

for every test function $w \in H(\operatorname{curl} ; \Omega)$. Here, the function space $H(\operatorname{curl} ; \Omega)$ is given by $\left\{\boldsymbol{w}: \boldsymbol{w} \in L_{2}(\Omega)\right.$ and $\left.\nabla \times \boldsymbol{w} \in L_{2}(\Omega)\right\}$ and we have

$$
\begin{aligned}
a(\boldsymbol{w}, \boldsymbol{H}) & =\int_{\Omega} \epsilon_{\mathrm{c}}^{-1}(\nabla \times \boldsymbol{w}) \cdot(\nabla \times \boldsymbol{H}) d \Omega \\
& -\int_{\Omega} \omega^{2} \mu_{0} \boldsymbol{w} \cdot \boldsymbol{H} d \Omega \\
& +\gamma \sum_{p=1}^{N_{\mathrm{p}}} \int_{\Gamma_{2}^{(p)}}(\hat{\boldsymbol{n}} \times \boldsymbol{w}) \cdot(\hat{\boldsymbol{n}} \times \boldsymbol{H}) d \Gamma, \\
b(\boldsymbol{w}) & =\sum_{p=1}^{N_{\mathrm{p}}} b_{p}(\boldsymbol{w})=-\sum_{p=1}^{N_{\mathrm{p}}} \int_{\Gamma_{2}^{(p)}} \boldsymbol{w} \cdot \boldsymbol{Q}^{(p)} d \Gamma .
\end{aligned}
$$

\subsubsection{Scattering parameters}

The scattering parameters [15] at waveguide ports $\Gamma_{2}^{(p)}$ can be expressed as follows

$$
S_{p q}^{\mathrm{r}}=\frac{V_{0 p}^{-}}{V_{0 q}^{+}}=\frac{E_{0 p}^{-}}{E_{0 q}^{+}}=\frac{E_{0 p}^{+}}{E_{0 q}^{+}} e^{-2 j k_{v} v^{(p)}}-\zeta b_{p}(\boldsymbol{H}),
$$

where $\zeta=\mu_{0} /\left(2 j k_{v} E_{0 p}^{+} E_{0 q}^{+} \int_{\Gamma_{2}^{(p)}}|\hat{\boldsymbol{n}} \times \boldsymbol{m}|^{2} d \Gamma\right)$ and $b_{p}(\cdot)$ is defined in Eq. (7). Here, the magnetic field $\boldsymbol{H}$ is the computed magnetic field for a non-zero incident wave associated with port $q$, with amplitude $E_{0 q}^{+}$for the electric field and $V_{0 q}^{+}$for the corresponding voltage. Also, the incident wave amplitude is zero for all the other ports and the amplitude $E_{0 p}^{-}$is associated with the electric field of the outward propagating wave for port $p$, where $V_{0 p}^{-}$is the corresponding voltage amplitude.

\subsection{System model}

The scattering parameters are measured at the coaxial cable ports of the adapters and, consequently, the adapters influence the measured signal. Below, we present a technique to estimate the scattering matrix $\mathbf{S}^{\mathrm{a}}$ of the adapters that connect the rectangular waveguides to the coaxial cables. Also, we provide a formula that transforms the scattering matrix $\mathbf{S}^{\mathrm{c}}$ measured at the coaxial cable ports of the adapters to the corresponding scattering matrix $\mathbf{S}^{\mathrm{r}}$ associated with the rectangular waveguide ports.

We represent the measurement system as illustrated in Fig. 3. The model involves the scattering matrix $\mathbf{S}^{\mathrm{r}}$ at the rectangular waveguide ports, as shown in Fig. 2, and it represents the rectangular waveguides and their intersection that yield the measurement region. Thus, $\mathbf{S}^{\mathrm{r}}$ can be directly computed by the $2 \mathrm{D}$ FEM as described above. Next, the measurement equipment shown in Fig. 1(a) allows for direct measurement of the scattering matrix $\mathbf{S}^{\mathrm{c}}$ associated with the coaxial cable ports of the adapters. (Before the measurement is performed, the network analyzer is calibrated up to the ports where the coaxial cables are connected to the adapters, where we use the standard SOLTcalibration - short, open, load and thru.) In order to relate $\mathbf{S}^{\mathrm{r}}$ to $\mathbf{S}^{\mathrm{c}}$, we need to characterize the adapters that connect each individual rectangular waveguide to its coaxial cable and we do that by means of a $2 \times 2$ scattering matrix

$$
\mathbf{S}^{\mathrm{a}}=\left[\begin{array}{ll}
S_{11}^{\mathrm{a}} & S_{12}^{\mathrm{a}} \\
S_{21}^{\mathrm{a}} & S_{22}^{\mathrm{a}}
\end{array}\right],
$$

where we assume that all adapters are identical. The (generalized) scattering parameters [16] in Eq. (9) are defined as $S_{p q}^{\text {a }}=$ $\sqrt{Z_{q} / Z_{p}}\left(V_{0 p}^{-} / V_{0 q}^{+}\right)$, where $Z_{p}$ is the characteristic impedance of the waveguide connected to port $p$ and the factor $\sqrt{Z_{q} / Z_{p}}$ preserves symmetry and makes the scattering matrix unitary for the lossless case.

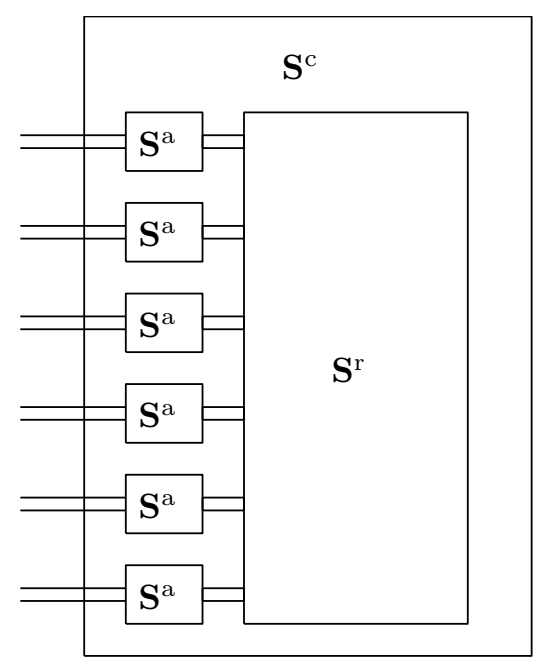

Figure 3: Model of the measurement equipment that is used for the calibration procedure.

Thus, the scattering matrix $\mathbf{S}^{\mathrm{c}}$ at the coaxial cable ports of the adapters may be expressed in terms of its constituents $\mathbf{S}^{\mathrm{r}}$ and $\mathbf{S}^{\mathrm{a}}$, which is illustrated in Fig. 3. At the rectangular waveguide ports, we have $\mathbf{u}^{\text {ro }}=\mathbf{S}^{\mathrm{r}} \mathbf{u}^{\text {ri }}$, where $\mathbf{u}^{\text {ro }}$ is voltage vector for the outward propagating waves and $\mathbf{u}^{\mathrm{ri}}$ is the voltage vector for the inward propagating waves in the rectangular waveguides shown in Fig. 2. In the corresponding manner, we define $\mathbf{u}^{\text {co }}$ as the outward propagating waves and $\mathbf{u}^{\mathrm{ci}}$ as the inward propagating waves on the coaxial-cable waveguides. According to Eq. (9) 
with $\left\{\mathbf{u}^{\mathrm{ci}}, \mathbf{u}^{\mathrm{ro}}\right\}$ incident on the adapters and $\left\{\mathbf{u}^{\mathrm{co}}, \mathbf{u}^{\mathrm{ri}}\right\}$ scattered from the adapters, we have

$$
\begin{aligned}
\mathbf{u}^{\mathrm{ro}} & =\left(\mathbf{u}^{\mathrm{co}}-S_{11}^{\mathrm{a}} \mathbf{u}^{\mathrm{ci}}\right) / S_{12}^{\mathrm{a}} \\
\mathbf{u}^{\mathrm{ri}} & =S_{21}^{\mathrm{a}} \mathbf{u}^{\mathrm{ci}}+S_{22}^{\mathrm{a}} \mathbf{u}^{\mathrm{ro}} \\
& =S_{21}^{\mathrm{a}} \mathbf{u}^{\mathrm{ci}}+S_{22}^{\mathrm{a}}\left(\mathbf{u}^{\mathrm{co}}-S_{11}^{\mathrm{a}} \mathbf{u}^{\mathrm{ci}}\right) / S_{12}^{\mathrm{a}}
\end{aligned}
$$

and these relations allows us to express $\mathbf{u}^{\text {ro }}=\mathbf{S}^{\mathrm{r}} \mathbf{u}^{\text {ri }}$ in terms of $\mathbf{u}^{\text {co }}$ and $\mathbf{u}^{\text {ci }}$. Thus, we have

$$
\mathbf{u}^{\mathrm{co}}=\left[\mathbf{I}-S_{22}^{\mathrm{a}} \mathbf{S}^{\mathrm{r}}\right]^{-1}\left[S_{11}^{\mathrm{a}} \mathbf{I}+\left(S_{12}^{\mathrm{a}} S_{21}^{\mathrm{a}}-S_{11}^{\mathrm{a}} S_{22}^{\mathrm{a}}\right) \mathbf{S}^{\mathrm{r}}\right] \mathbf{u}^{\mathrm{ci}}
$$

where $\mathbf{I}$ is the identity matrix of the same size as $\mathbf{S}^{\mathrm{r}}$. Consequently, we have the relation $\mathbf{u}^{\text {co }}=\mathbf{S}^{\mathrm{c}} \mathbf{u}^{\text {ci }}$ with $\mathbf{S}^{\mathrm{c}}$ expressed in terms of $\mathbf{S}^{\mathrm{r}}$ and $\mathbf{S}^{\mathrm{a}}$ and this gives the matrix relation

$$
\xi_{1} \mathbf{I}+\xi_{2} \mathbf{S}^{\mathrm{r}} \mathbf{S}^{\mathrm{c}}+\xi_{3} \mathbf{S}^{\mathrm{r}}=\mathbf{S}^{\mathrm{c}}
$$

where we have the coefficients $\xi_{1}=S_{11}^{\mathrm{a}}, \xi_{2}=S_{22}^{\mathrm{a}}$ and $\xi_{3}=$ $S_{12}^{\mathrm{a}} S_{21}^{\mathrm{a}}-S_{11}^{\mathrm{a}} S_{22}^{\mathrm{a}}$.

Next, we measure $\mathbf{S}^{\mathrm{c}}=\mathbf{S}_{0}^{\mathrm{c}}$ and compute $\mathbf{S}^{\mathbf{r}}=\mathbf{S}_{0}^{\mathrm{r}}$, where the sub-index naught indicates that the measurement region is empty in the sense that it only contains air. Then, we determine the parameters $\left(\xi_{1}, \xi_{2}, \xi_{3}\right)$ by solving the over-determined system of linear equations (10) in the least-squares sense, which is repeated on a frequency-by-frequency basis from $2.7 \mathrm{GHz}$ to 5.1 GHz.

For a measured scattering matrix $\overline{\mathbf{S}^{\mathbf{c}}}$ with a sample placed in the measurement equipment, we can compute the corresponding scattering matrix $\overline{\mathbf{S}^{\mathrm{r}}}$ at the ports of the rectangular waveguides, i.e. after compensation for the adapters, by means of the relation

$$
\overline{\mathbf{S}^{\mathrm{r}}}=\left[\overline{\mathbf{S}^{\mathrm{c}}}-\xi_{1} \mathbf{I}\right]\left[\xi_{2} \overline{\mathbf{S}^{\mathrm{c}}}+\xi_{3} \mathbf{I}\right]^{-1},
$$

which can be used in order to determine the permittivity of a sample placed in the measurement region.

\section{Reconstruction algorithm}

The inverse scattering problem deals with the estimation of the complex permittivity in the measurement region. In detail, the reconstruction of the complex permittivity $\epsilon_{\mathrm{c}}=\epsilon_{\mathrm{c}}(\boldsymbol{r}, \omega ; \mathbf{p})$ is performed by minimizing a goal function $g\left(\epsilon_{\mathrm{c}}\right)$ with respect to the parameter vector $\mathbf{p}$, that describes the space $\boldsymbol{r}$ and frequency $\omega$ variation of the permittivity in the measurement region. Thus, we solve the optimization problem

$$
\begin{aligned}
& \min _{\mathbf{p}} g\left(\epsilon_{\mathrm{c}}(\boldsymbol{r}, \omega ; \mathbf{p})\right) \\
& \text { s.t. } \operatorname{Im}\left\{\omega \epsilon_{\mathrm{c}}(\boldsymbol{r}, \omega ; \mathbf{p})\right\}<0 .
\end{aligned}
$$

This is a nonlinear constrained optimization problem and we solve it by means of the sparse nonlinear solver (SNOPT) implemented in TOMLAB [17].

The goal function $g\left(\epsilon_{\mathrm{c}}\right)$ is expressed as the root-mean-square (RMS) value of the misfit between the computed $\mathbf{S}^{\mathrm{r}}\left(\omega ; \epsilon_{\mathrm{c}}\right)$ and the measured $\overline{\mathbf{S}^{r}}(\omega)$ scattering parameters, averaged over the number of ports $N_{\mathrm{p}}$ and the frequency points in the range $\left(\omega_{\mathrm{L}}, \omega_{\mathrm{U}}\right)$. Thus, we have

$$
g\left(\epsilon_{\mathrm{c}}\right)=\left\|\mathbf{S}^{\mathrm{r}}\left(\omega ; \epsilon_{\mathrm{c}}\right)-\overline{\mathbf{S}^{\mathrm{r}}}(\omega)\right\|,
$$

where

$$
\|\mathbf{S}\|=\left[\frac{1}{N_{\mathrm{p}}^{2}} \sum_{p=1}^{N_{\mathrm{p}}} \sum_{q=1}^{N_{\mathrm{p}}} \frac{1}{\omega_{\mathrm{U}}-\omega_{\mathrm{L}}} \int_{\omega_{\mathrm{L}}}^{\omega_{\mathrm{U}}}\left|S_{p q}\right|^{2} d \omega\right]^{1 / 2}
$$

and the indices $p$ and $q$ correspond to rows and columns, respectively, in the scattering matrix. It should be noticed that this norm also incorporates the diagonal elements of the scattering matrix and, consequently, our measurement procedure combines transmission measurements with reflection measurements.

The minimization is performed subject to the constraint $\operatorname{Im}\left\{\omega \epsilon_{\mathrm{c}}(\boldsymbol{r}, \omega ; \mathbf{p})\right\}<0$, which enforces a passive material. Here, this constraint is applied for all frequencies and spatial locations that are involved in the estimation of $\epsilon_{\mathrm{c}}$.

\subsection{Sensitivity analysis}

The first-order variation of the goal function $g\left(\epsilon_{\mathrm{c}}\right)$ with respect to a perturbation $\delta \epsilon_{\mathrm{c}}$ in the material permittivity $\epsilon_{\mathrm{c}}$ is computed using the first-order variation of the scattering parameters $\delta S_{p q}^{\mathrm{r}}$.

Let the original permittivity $\epsilon_{\mathrm{c}}$ be perturbed by $\delta \epsilon_{\mathrm{c}}$. This yields a perturbation $\delta \boldsymbol{H}$ in the original magnetic field $\boldsymbol{H}$ and, thus, a variation in the scattering parameters (8). This corresponds to the first-order variation of the scattering parameters of $\delta S_{p q}^{\mathrm{r}}=-\zeta b_{p}(\delta \boldsymbol{H})=\zeta \delta a\left(\boldsymbol{H}_{\mathrm{adj}}, \boldsymbol{H}_{\text {orig }}\right)[18]$ and it yields

$$
\begin{aligned}
\delta S_{p q}^{\mathrm{r}}= & -\frac{\mu_{0}}{j w k_{v} E_{0 p, \text { adj }}^{+} E_{0 q, \text { orig }}^{+}} \\
& \int_{\Omega} \frac{\delta \epsilon_{\mathrm{c}}}{\epsilon_{\mathrm{c}}^{2}}\left(\nabla \times \boldsymbol{H}_{\mathrm{adj}}\right) \cdot\left(\nabla \times \boldsymbol{H}_{\text {orig }}\right) d \Omega,
\end{aligned}
$$

where $w$ is the waveguide width, $E_{0 p \text {,adj }}^{+}$represents the amplitude of the excitation for the adjoint field problem $\boldsymbol{H}_{\text {adj }}$ at port $p$ and $E_{0 q \text {,orig }}^{+}$is the amplitude of the excitation for the original field problem $\boldsymbol{H}_{\text {orig }}$ at port $q$.

\subsection{Material models}

Commonly, the formulation of an inverse scattering problem involves one or more material parameters associated with each computational cell as described in [19], [20] and [21]. This implies that the number of degrees of freedom in the reconstruction is related to the number of elements exploited to discretize the field problem. Consequently, refinements in the discretization, in order to improve the accuracy of the field solution, yields an increase in the number of unknowns in the reconstruction. Thus, the reconstruction problem tends to become more ill-conditioned, which prompts for regularization.

Here, we choose an alternative route, where we represent the permittivity distribution by means of a parameterization. The parameterization is independent of the computational mesh, so the number of degrees of freedom in the reconstruction problem is not influenced by refinements in the computational mesh. 


\subsubsection{Spatial representation}

The spatial variation of the permittivity is represented by a set of local basis functions $\varphi_{k}(\boldsymbol{r})$ and, in this article, we use piecewise constant basis functions. However, it is easy to make other choices such as piecewise linear or quadratic polynomials, should it be beneficial for the application at hand. Thus, the complex permittivity is parameterized as

$$
\epsilon_{\mathrm{c}}(\boldsymbol{r}, \omega ; \mathbf{p})=\epsilon_{0} \sum_{k=1}^{K} a_{k}\left(\omega ; \mathbf{p}_{k}\right) \varphi_{k}(\boldsymbol{r}),
$$

where $\epsilon_{0}$ is the permittivity of vacuum and $\mathbf{p}$ is the global parameter vector. Here, the coefficients $a_{k}\left(\omega ; \mathbf{p}_{k}\right)$ describe the frequency dependence of the permittivity associated with the $k$-th basis function. Moreover, these coefficients also depend on a set of unknown local parameters $\mathbf{p}_{k}$ that influence the frequency variation of the permittivity. The global parameter vector $\mathbf{p}=\left[\mathbf{p}_{1}, \mathbf{p}_{2}, \ldots, \mathbf{p}_{K}\right]$ is composed by these local parameter vectors $\mathbf{p}_{k}$.

\subsubsection{Frequency response}

We consider media that may be described by the HavriliakNegami response [22], which can expressed mathematically as

$$
\epsilon_{\mathrm{c}}(\boldsymbol{r}, \omega)=\epsilon_{0}\left[\epsilon_{\infty}(\boldsymbol{r})+\frac{\epsilon_{\mathrm{s}}(\boldsymbol{r})-\epsilon_{\infty}(\boldsymbol{r})}{\left[1+(j \omega \tau(\boldsymbol{r}))^{\alpha}\right]^{\beta}}\right],
$$

where $\epsilon_{\mathrm{S}}$ is the static permittivity, $\epsilon_{\infty}$ is the optical permittivity and $\tau$ is the relaxation time. Thus, the coefficients $a_{k}\left(\omega ; \mathbf{p}_{k}\right)$ in the parameterization are given by

$$
a_{k}\left(\omega ; \mathbf{p}_{k}\right)=p_{k, 2}+\frac{p_{k, 1}-p_{k, 2}}{\left[1+\left(j \omega p_{k, 3}\right)^{p_{k, 4}}\right]^{p_{k, 5}}}
$$

where $p_{k, 1}, p_{k, 2}, p_{k, 3}, p_{k, 4}$ and $p_{k, 5}$ correspond to $\epsilon_{\mathrm{s}}, \epsilon_{\infty}, \tau, \alpha$ and $\beta$, respectively.

We identify three special cases

- Debye media: $\alpha=\beta=1$

- Cole-Cole media: $0<\alpha<1$ and $\beta=1$

- Cole-Davidson media: $\alpha=1$ and $0<\beta<1$

It is easy to prove that the Debye and the Cole-Cole media fulfills the constraint (13), where the frequency is assumed to be non-zero. In addition, we note that the Maxwell-Garnett mixing formula [23] yields an effective permittivity that can be expressed as a Debye medium if the mixture consists of (i) air and (ii) inclusion particles that have a permittivity described by a Debye model.

\subsection{Estimation of uncertainties}

Given a dielectric sample that is placed in the measurement region, we attempt to measure, e.g., its size and position by means of other (conventional) techniques such as calipers and rulers. These measurement results are described by a parameter vector $\tilde{\mathbf{p}}$ and it is used as input for the optimization problem (12)-(13), which features an underlying field problem with the dielectric sample described in terms of its (known) size and position. Thus, uncertainties $\delta \tilde{\mathbf{p}}$ in the a priori measured parameters $\tilde{\mathbf{p}}$ yield uncertainties $\delta \mathbf{p}$ in the estimated permittivity model described by $\mathbf{p}$. Now, we wish to exploit the sensitivities presented above to establish a relation between $\delta \tilde{\mathbf{p}}$ and $\delta \mathbf{p}$. (In the following, we assume that the uncertainties $\delta \tilde{\mathbf{p}}$ are uncorrelated.) The sensitivity with respect to the permittivity is shown in Eq. (16) and the corresponding results for shape perturbations (or displacements) of the sample can be derived with the approach presented in Ref. [24].

First, we introduce the vectorized scattering matrix $\mathbf{s}=$ $\operatorname{vec}(\mathbf{S})$. The model of the system is linearized around a linearization point $\left\{\mathbf{p}_{0}, \tilde{\mathbf{p}}_{0}\right\}$, which yields

$$
\mathbf{s}=\mathbf{s}_{0}+\left(\nabla_{\mathbf{p}} \mathbf{s}\right) \delta \mathbf{p}+\left(\nabla_{\tilde{\mathbf{p}}} \mathbf{s}\right) \delta \tilde{\mathbf{p}}+\ldots
$$

Here, $\mathbf{s}_{0}$ is the model evaluated at the linearization point $\left\{\mathbf{p}_{0}, \tilde{\mathbf{p}}_{0}\right\}$. Similarly, $\left(\nabla_{\mathbf{p}} \mathbf{s}\right)$ and $\left(\nabla_{\tilde{\mathbf{p}}} \mathbf{s}\right)$ are the sensitivities with respect to $\mathbf{p}$ and $\tilde{\mathbf{p}}$, respectively, evaluated at the linearization point $\left\{\mathbf{p}_{0}, \tilde{\mathbf{p}}_{0}\right\}$. Also, we express the measurement result as $\overline{\mathbf{s}}=\mathbf{s}_{0}+\delta \overline{\mathbf{s}}$, where $\delta \overline{\mathbf{s}}$ describes the deviation between the measurement and the model evaluated at the linearization point. We use $\delta \overline{\mathbf{s}}$ in an attempt to quantify discrepancies that can not be described by the model as $\mathbf{p}$ and $\tilde{\mathbf{p}}$ are varied.

At the optimum, we have the necessary condition $\nabla_{\mathbf{p}} g(\mathbf{p})=$ 0 . Now, we let the linearization point $\left\{\mathbf{p}_{0}, \tilde{\mathbf{p}}_{0}\right\}$ coincide with the optimum and this gives the approximate relation

$$
\begin{aligned}
\delta \mathbf{p}= & -\left[\left(\nabla_{\mathbf{p}} \mathbf{s}\right)^{\mathrm{H}}\left(\nabla_{\mathbf{p}} \mathbf{s}\right)\right]^{-1} \operatorname{Re}\left\{\left(\nabla_{\mathbf{p}} \mathbf{s}\right)^{\mathrm{H}}\left(\nabla_{\tilde{\mathbf{p}}} \mathbf{s}\right)\right\} \delta \tilde{\mathbf{p}} \\
& -\left[\left(\nabla_{\mathbf{p}} \mathbf{s}\right)^{\mathrm{H}}\left(\nabla_{\mathbf{p}} \mathbf{s}\right)\right]^{-1} \operatorname{Re}\left\{\left(\nabla_{\mathbf{p}} \mathbf{s}\right)^{\mathrm{H}} \delta \overline{\mathbf{s}}\right\},
\end{aligned}
$$

where higher-order terms in Eq. (20) have been neglected.

\section{Results}

First, we present the characteristic features of our estimation of the scattering matrix for the adapters. Then, we estimate the dielectric constant for a set of acrylic-glass rods, which is compared to a conventional cavity resonance measurement. Next, we estimate the permittivity of a plastic holder, which is used in the final measurements applied to a mixture of air and moist microcrystalline cellulose (MCC) pellets.

\subsection{Empty cavity}

The first series of measurements is performed for the empty cavity with only air in the measurement region, which is indicated by the dashed circle in Fig. 2. Given the measured response of the empty measurement system, we estimate the scattering parameters in Eq. (9) based on Eq. (10). The scattering parameters are shown in Fig. 4 in terms of their magnitude.

Next, we assess the agreement between the model and the measurements based on the residual

$$
r=\left[\frac{1}{N_{\mathrm{p}}^{2}} \sum_{p=1}^{N_{\mathrm{p}}} \sum_{q=1}^{N_{\mathrm{p}}}\left|S_{p q}^{\mathrm{r}}-\overline{S_{p q}^{\mathrm{r}}}\right|^{2}\right]^{1 / 2},
$$




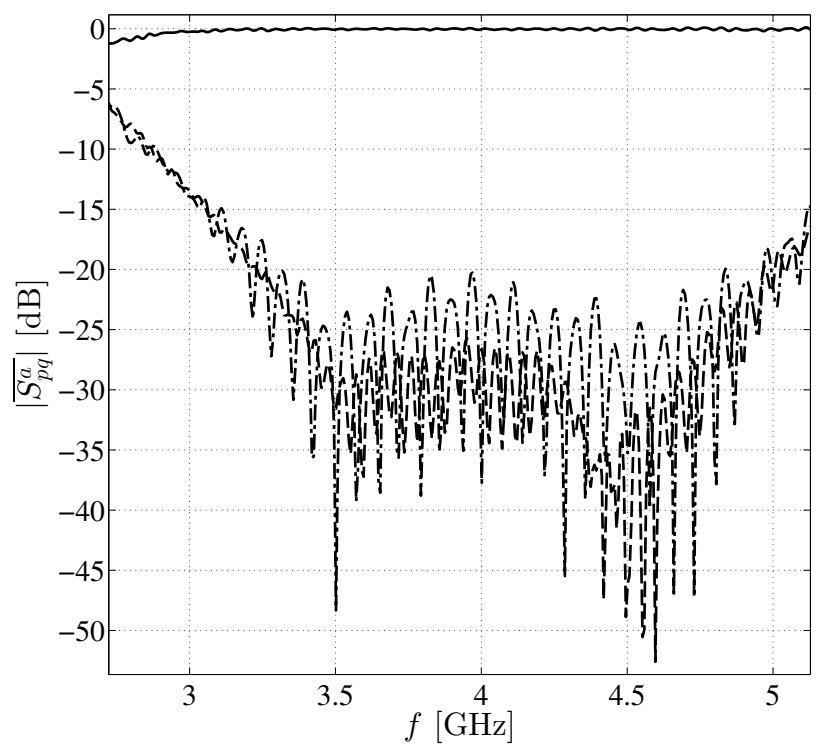

Figure 4: Estimated magnitude of the scattering parameters for the adapters as a function of frequency: solid curve $-\left|S_{12}^{\mathrm{a}}\right|=\left|S_{21}^{\mathrm{a}}\right|$; dashed curve $-\left|S_{11}^{\mathrm{a}}\right|$; and dash-dotted curve $-\left|S_{22}^{\mathrm{a}}\right|$.

which is formed by comparing the model $\mathbf{S}^{\mathrm{r}}$ in Fig. 3 with the measured response $\overline{\mathbf{S}^{\mathrm{r}}}$ of the system. In Fig. 5, the solid curve shows the residual as a function of the frequency and we find that the residual $r$ is less than $-30 \mathrm{~dB}$ for basically the entire frequency band from $2.7 \mathrm{GHz}$ to $5.1 \mathrm{GHz}$. This demonstrates that $\overline{\mathbf{S}^{\mathrm{r}}}$ computed from Eq. (11) is in good agreement with the model $\mathbf{S}^{\mathrm{r}}$. Next, we perform a sequence of measurements that involves disassembling and reassembling the measurement equipment, where each measurement is performed for the empty system. Thus, this operation corresponds to the procedure necessary for placing a dielectric sample subject to measurement inside the measurement system. In Fig. 5, the dashed curve shows the residual as a function of the frequency for the empty measurement region (after opening and closing the measurement equipment) and we note that the residual is maintained at the same low level of about $-30 \mathrm{~dB}$ for the frequency band of interest. Thus, we conclude that the measurement equipment is stable in the sense that the measurements for the empty measurement system are reproducible.

\subsection{Dielectric cylinder}

For testing purposes, we use a measurement on a dielectric cylinder of acrylic glass, where the diameter of the cylinder is $10.4 \mathrm{~mm}$ and its height equals the height of the measurement region. The dielectric constant for the acrylic glass is measured in a circular cylinder cavity $[12,25]$ and the relative permittivity is $2.62 \pm 0.09$ based on the $\mathrm{TM}_{010}$ mode with the resonance frequency at $918 \mathrm{MHz}$. (It should be noted that this value is measured at a substantially lower frequency as compared to the measurement system presented in this article, which operates in the frequency band from $2.7 \mathrm{GHz}$ to $5.1 \mathrm{GHz}$.) In addition, for acrylic-glass sheets, Afzalzadeh reports in [26] a relative permittivity of $2.55 \pm 0.13$ at $8 \mathrm{GHz}$ and Dudeck et al. reports in [27] a value of $2.62 \pm 0.04$ at $20 \mathrm{GHz}$.

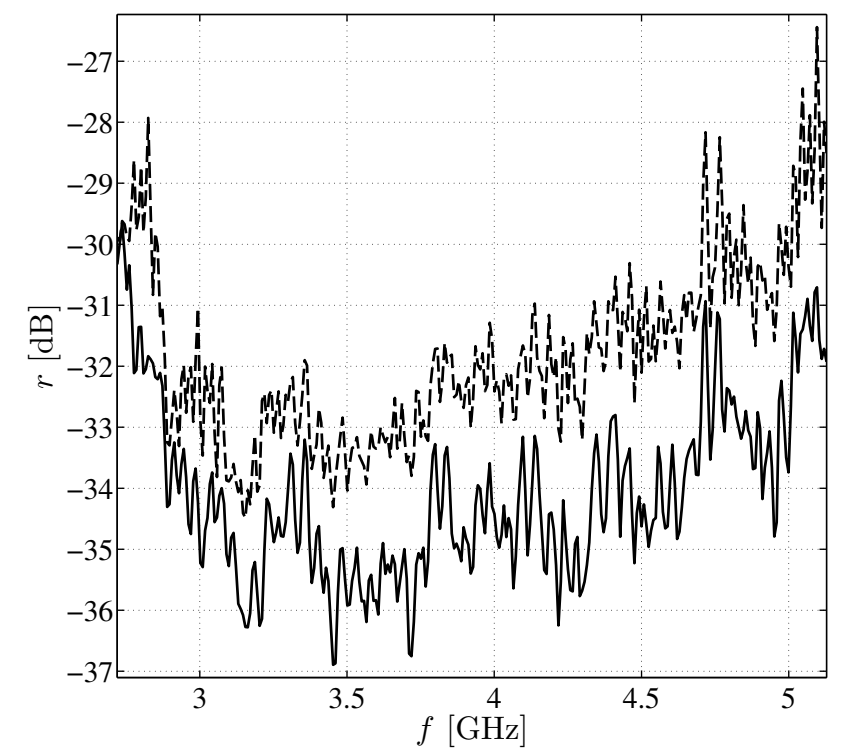

Figure 5: Residual between the computational model $\mathbf{S}^{\mathrm{r}}$ and the measured response $\overline{\mathbf{S}^{r}}$ for the empty measurement system: solid curve - residual for the measurement used to determine $\mathbf{S}^{\mathrm{a}}$; and dashed curve - measurement after disassembling and reassembling of the measurement equipment.

We placed one, three and seven samples of the acrylic-glass cylinder at the center of the measurement domain as shown in Fig. 6. Given the measured scattering parameters, the goal function (14) between the computational model and the measurement is shown in Fig. 7 as a function of the permittivity $\epsilon_{\mathrm{r}}$ $\left(=\epsilon_{\mathrm{s}}=\epsilon_{\infty}\right.$ in Eq. (18)), where the geometry of the acrylic-glass samples is considered known in the computational model. We notice a clear minimum with respect to the permittivity at 2.54 for all three configurations of the acrylic-glass cylinders.

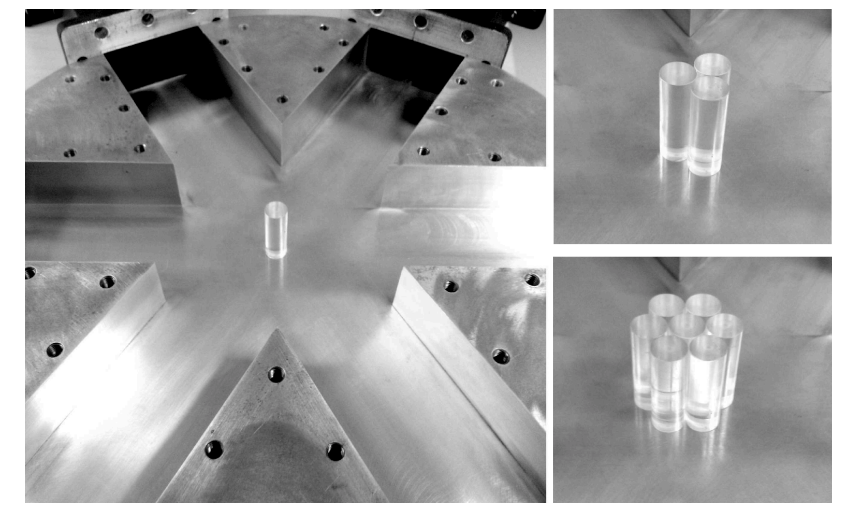

Figure 6: Three configurations with one, three and seven acrylic-glass cylinders.

We estimate that the positioning uncertainty is $\delta \tilde{x}_{\mathrm{c}}=\delta \tilde{y}_{\mathrm{c}}=$ $\pm 1 \mathrm{~mm}$ and that the uncertainty in the radius of the dielectric cylinder is $\delta \tilde{a}= \pm 0.1 \mathrm{~mm}$. Next, we use the procedure described in Sec. 3.3 to estimate the uncertainty in the permittivity of the sample given estimates on the uncertainties in its size 


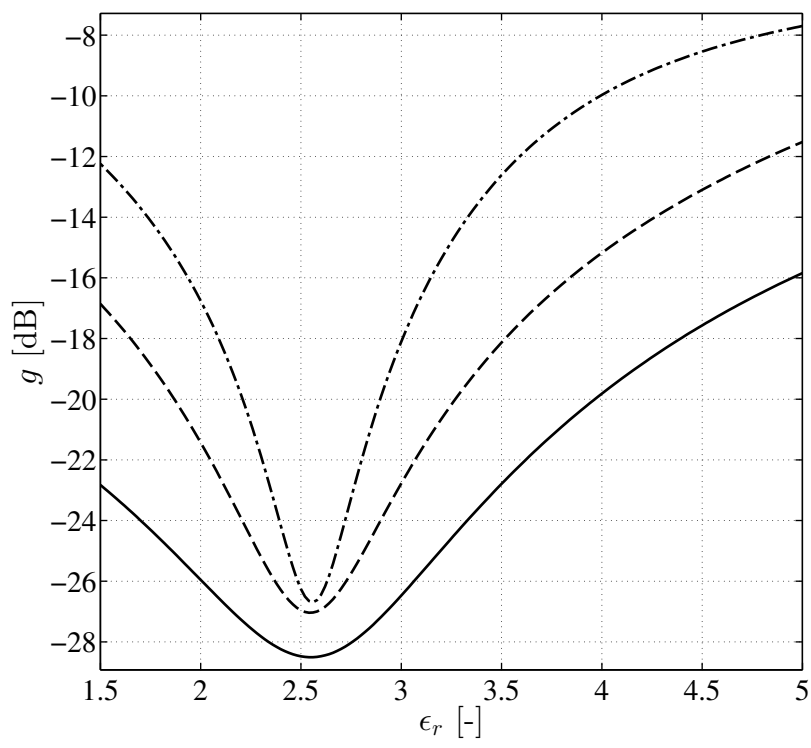

Figure 7: Misfit between the computational model and the measurement for three configurations with acrylic-glass cylinder: solid curve - one acrylic-glass cylinder; dashed curve - three acrylic-glass cylinders; and dash-dotted curve seven acrylic-glass cylinders.

and position, which yields

$$
\begin{aligned}
\delta \epsilon_{\mathrm{r}} & =\mathfrak{s}_{\tilde{x}_{\mathrm{c}}} \delta \tilde{x}_{\mathrm{c}}+\mathfrak{s}_{\tilde{y}_{\mathrm{c}}} \delta \tilde{y}_{\mathrm{c}}+\mathfrak{s}_{\tilde{a}} \delta \tilde{a}+\delta \overline{\mathfrak{s}}, \\
\mathfrak{s}_{\tilde{x}_{\mathrm{c}}} & =-\frac{\operatorname{Re}\left\{\left(\partial \mathbf{s} / \partial \epsilon_{\mathrm{r}}\right)^{\mathrm{H}}\left(\partial \mathbf{s} / \partial \tilde{x}_{\mathrm{c}}\right)\right\}}{\left(\partial \mathbf{s} / \partial \epsilon_{\mathrm{r}}\right)^{\mathrm{H}}\left(\partial \mathbf{s} / \partial \epsilon_{\mathrm{r}}\right)}, \\
\mathfrak{s}_{\tilde{y}_{\mathrm{c}}} & =-\frac{\operatorname{Re}\left\{\left(\partial \mathbf{s} / \partial \epsilon_{\mathrm{r}}\right)^{\mathrm{H}}\left(\partial \mathbf{s} / \partial \tilde{y}_{\mathrm{c}}\right)\right\}}{\left(\partial \mathbf{s} / \partial \epsilon_{\mathrm{r}}\right)^{\mathrm{H}}\left(\partial \mathbf{s} / \partial \epsilon_{\mathrm{r}}\right)}, \\
\mathfrak{s}_{\tilde{a}} & =-\frac{\operatorname{Re}\left\{\left(\partial \mathbf{s} / \partial \epsilon_{\mathrm{r}}\right)^{\mathrm{H}}(\partial \mathbf{s} / \partial \tilde{a})\right\}}{\left(\partial \mathbf{s} / \partial \epsilon_{\mathrm{r}}\right)^{\mathrm{H}}\left(\partial \mathbf{s} / \partial \epsilon_{\mathrm{r}}\right)}, \\
\delta \overline{\mathfrak{s}} & =-\frac{\operatorname{Re}\left\{\left(\partial \mathbf{s} / \partial \epsilon_{\mathrm{r}}\right)^{\mathrm{H}}(\delta \overline{\mathbf{s}})\right\}}{\left(\partial \mathbf{s} / \partial \epsilon_{\mathrm{r}}\right)^{\mathrm{H}}\left(\partial \mathbf{s} / \partial \epsilon_{\mathrm{r}}\right)},
\end{aligned}
$$

where the coefficients $\mathfrak{s}_{\tilde{x}_{\mathfrak{c}}}, \mathfrak{s}_{\tilde{y}_{\mathrm{c}}}$ and $\mathfrak{s}_{\tilde{a}}$ are evaluated by means of the FEM and the values are given in Tab. 1. According to Tab. 1 , it is clear that the sensitivity $\mathfrak{s}_{\tilde{a}}$ associated with the acrylic-glass cylinder radius is many orders of magnitude larger than the sensitivities $\left(\mathfrak{s}_{\tilde{x}_{\mathrm{c}}}, \mathfrak{s}_{\tilde{y}_{\mathrm{c}}}\right)$ associated with the position of the cylinder. This is a consequence of the derivation of Eq. (23), the symmetry of the experiment and that the sample is placed at the center of the measurement region. A more careful investigation based on direct computation with an exhaustive parameter study (that incorporates the higher-order terms not included in Eq. (23)) reveals that a positioning uncertainty of $\delta \tilde{x}_{\mathrm{c}}=\delta \tilde{y}_{\mathrm{c}}= \pm 1 \mathrm{~mm}$ yields a corresponding uncertainty in the permittivity of $\delta \epsilon_{\mathrm{r}}= \pm 0.008$. This yields an uncertainty $\delta \epsilon_{\mathrm{r}}= \pm 0.06$ for the permittivity, where we also included an estimate of the unpredictable deviations $\delta \bar{s} \simeq 0.03$ in the scattering parameters based on the disassembling and reassembling of the empty measurement cavity shown in Fig. 5. Thus, we conclude that $\epsilon_{\mathrm{r}}=2.54 \pm 0.06$, which corresponds to a measurement accuracy of better than $3 \%$ given that the uncertainty contributions are added in the root-mean-square sense.
Table 1: Sensitivity of the permittivity with respect to different parameters $\tilde{p}$, where the parameters are the position $\left(\tilde{x}_{\mathrm{c}}, \tilde{y}_{\mathrm{c}}\right)$ and radius $\tilde{a}$ for a circular dielectric cylinder.

\begin{tabular}{llll}
\hline$\tilde{p}$ & $\mathfrak{s}_{\tilde{p}}\left[\mathrm{~m}^{-1}\right]$ & $\delta \tilde{p}[\mathrm{~m}]$ & $\left|\mathfrak{s}_{\tilde{p}} \delta \tilde{p}\right|[-]$ \\
\hline$\tilde{x}_{\mathrm{c}}$ & $-9 \cdot 10^{-4}$ & $10^{-3}$ & $9 \cdot 10^{-7}$ \\
$\tilde{y}_{\mathrm{c}}$ & $+6 \cdot 10^{-7}$ & $10^{-3}$ & $6 \cdot 10^{-10}$ \\
$\tilde{a}$ & $+5 \cdot 10^{+2}$ & $10^{-4}$ & $5 \cdot 10^{-2}$ \\
\hline
\end{tabular}

\subsubsection{Replicability with respect to noise level}

Figure 8 shows the estimated permittivity of the single acrylic-glass cylinder as a function of the signal-to-noise ratio (SNR). Here, we have added numerically generated white Gaussian noise to the measurement data and performed 100 different realizations for each SNR to generate the statistics. It is clear that the reconstruction algorithm produces replicable estimates of the relative permittivity within the measurement accuracy for an $\mathrm{SNR}$ of $20 \mathrm{~dB}$ or greater.

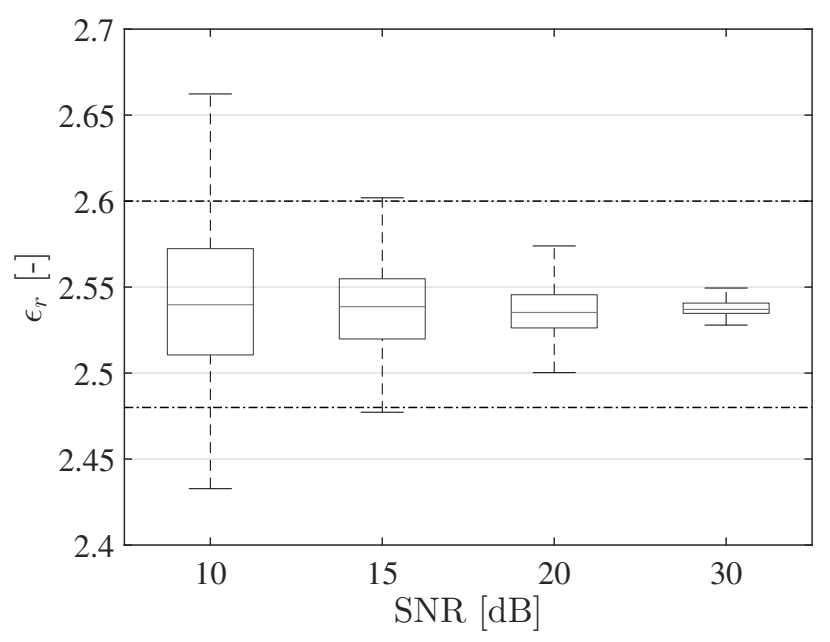

Figure 8: Boxplot showing the statistics of the estimated relative permittivity for a single acrylic-glass cylinder versus SNR. The median, 25th and 75th percentile are indicated by the box and the whiskers extend to cover approximately $99.3 \%$ of the data points. The horizontal dashed-dotted curves show the measurement accuracy estimated from the uncertainty in the sample radius, position and the disassembling and reassembling of the measurement system.

\subsection{Microcrystalline cellulose}

The results for the acrylic-glass cylinder are encouraging and, next, we wish to measure the effective permittivity of densely packed moist pellets of MCC, where air is the medium between the spherical pellets. In order to keep the pellets in place, we use the plastic holder shown in Fig. 9(a) together with a sealing plastic film. First, we incorporate the holder in our model of the measurement system for the frequency band of interest.

\subsubsection{Characterization of holder}

Despite the fact that the holder is a 3D object, we attempt to model it with our FEM program that is intended for $2 \mathrm{D}$ 


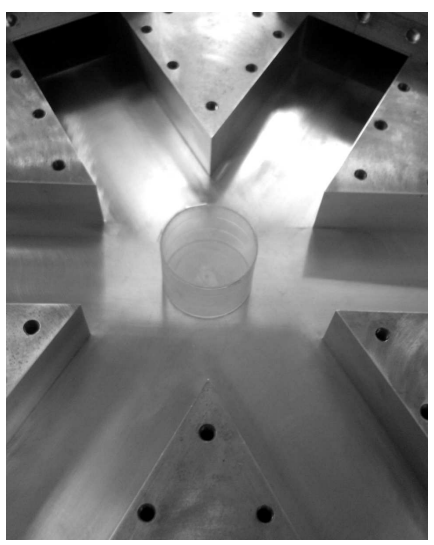

(a) Empty holder.

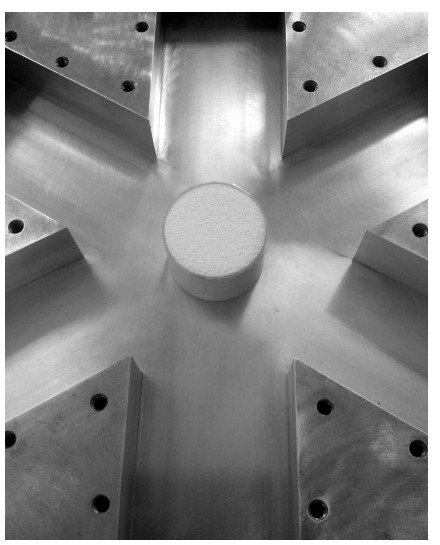

(b) Holder with MCC.
Figure 9: Plastic holder with the sealing plastic film: (a) empty holder used for calibration; and (b) holder with MCC pellets.

problems, which would preserve the computational advantages that we experienced for the acrylic-glass cylinders. The plastic holder is placed in the measurement region without the plastic film as shown in Fig. 9(a). The height of the holder's wall is equal to the height of the measurement cavity. In the computational model for 2D problems, the annular region $(19.8 \mathrm{~mm}$ $<r<20.7 \mathrm{~mm}$ in polar coordinates) is occupied by the wall of the holder and it is assigned the permittivity $\epsilon_{0} \epsilon_{\mathrm{h}}$ and the surrounding air has the permittivity $\epsilon_{0}$. For the circular region ( $r<19.8 \mathrm{~mm}$ in polar coordinates) that resides inside the holder's wall, we have the plastic bottom part of the holder in combination with the air above the bottom. For a vertical electric field, the effective permittivity $\epsilon_{0} \epsilon_{\text {eff }}$ obeys the relation $\left\langle D_{z}\right\rangle=\epsilon_{0} \epsilon_{\mathrm{eff}}\left\langle E_{z}>\right.$, where we have the average displacement field $\left\langle D_{z}\right\rangle$ and the average electric field $\left\langle E_{z}\right\rangle$. This relation yields the effective permittivity

$$
\epsilon_{\mathrm{eff}}=\frac{d_{0}+d_{\mathrm{h}}}{d_{0}+d_{\mathrm{h}} / \epsilon_{\mathrm{h}}}
$$

where $d_{0}=28 \mathrm{~mm}$ is the height occupied by the air and $d_{\mathrm{h}}=$ $1 \mathrm{~mm}$ is the thickness of the bottom part of the plastic holder. Given the physical dimensions and position of the holder (measured by calipers and rulers) and the measured scattering parameters, our gradient-based optimization method yields $\epsilon_{\mathrm{h}}=$ $2.3 \pm 0.3$. Here, we use the procedure described in Sec. 3.3 to estimate the uncertainty in the permittivity.

The main purpose of the holder is to act as a container for the MCC pellets. However, we also would like to perform the measurements on the MCC pellets for different amounts of moisture content and, consequently, it is desirable that the holder also maintains the moisture content of the pellets during the measurement. In an attempt to achieve this objective, we seal the opening of the holder with a plastic film. The plastic film is indeed very thin and, as a consequence of its very low mass, it does not influence the scattering parameters in any substantial manner.

Given the estimated permittivity for the holder, the solid curve in Fig. 10 shows the residual as a function of frequency, which is again about $-30 \mathrm{~dB}$ for the frequency band of interest. We conclude that the computational model with the (homogenized) holder together with the thin plastic film agrees well with the measured response. For comparative purposes, the dashed curve in Fig. 10 shows the residual of the measurement with the holder as compared to the computational model without the holder being present, i.e. the measurement region is empty. Clearly, the incorporation of the holder in the 2D FEM is successful and the residual is maintained at about as low levels as previously demonstrated in Fig. 5.

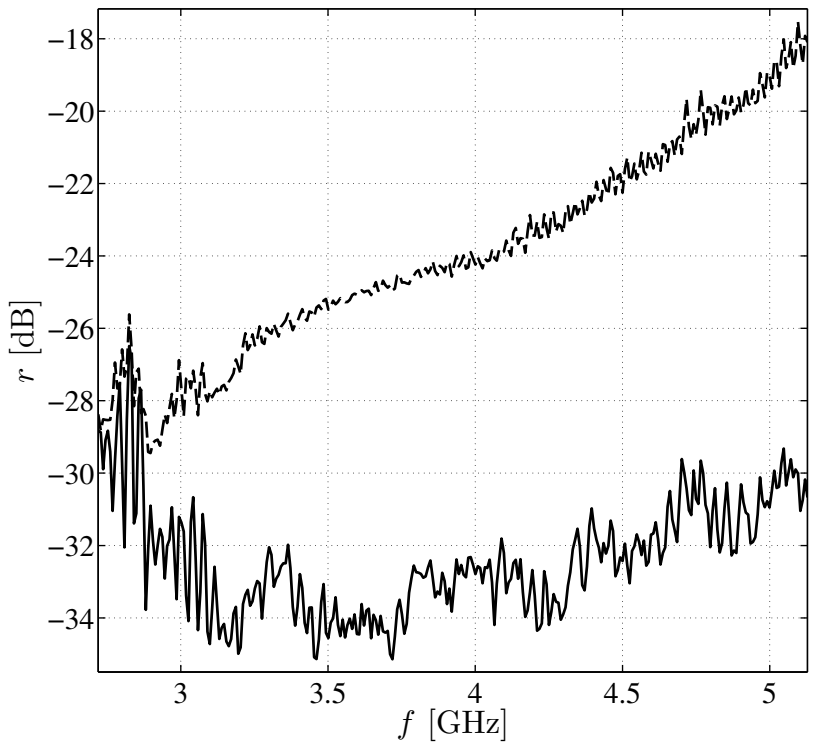

Figure 10: Residual for plastic holder with the sealing plastic film: solid curve FEM model that incorporates the holder with the permittivity $\epsilon_{0} \epsilon_{\mathrm{h}}$; and dashed curve - FEM model without the holder.

\subsubsection{Microcrystalline cellulose}

Finally, we measure the effective permittivity of densely packed MCC pellets with different amounts of moisture content. The pellets are moisturized and stored in sealed bags for about one week in order to allow the moisture to distribute uniformly among and fully diffuse into the pellets. Four sample sets of MCC pellets with different levels of moisture content are prepared. The moisture content is measured for the different sample sets by means of a loss-on-drying instrument and the results are shown in Tab. 2 .

Table 2: Sample set labels for MCC pellets and measured value for the moisture content, where the values are given in weight percent.

\begin{tabular}{rllll} 
Set & A & B & C & D \\
\hline Moisture $[\%(w / w)]$ & 9.2 & 12.2 & 16.8 & 22.8 \\
\hline
\end{tabular}

Next, we place $31 \pm 0.8 \mathrm{~g}$ of the pellets from a particular sample set in the plastic holder and the plastic film is used to seal the holder. The holder with the MCC pellets is placed at the center of the measurement region and the scattering parameters are measured from $2.7 \mathrm{GHz}$ to $5.1 \mathrm{GHz}$. The sample and 
the measurement equipment are in thermal equilibrium with the room, where the temperature is measured to be $23^{\circ} \mathrm{C}$.

Table 3 shows the estimated parameters $\epsilon_{\mathrm{s}}, \epsilon_{\infty}$ and $\tau$ that describe a Debye model of the effective permittivity for the mixture of moist pellets and air. Here, we use the procedure described in Sec. 3.3 to estimate the uncertainty in the Debye parameters given uncertainties in the position, size and permittivity of the holder. The permittivity $\epsilon_{\mathrm{s}}$ in the static limit increases with moisture content, whereas the relaxation time $\tau$ decreases. Also, it is noticed that the parameter $\epsilon_{\infty}$ does not depend monotonically on the moisture content. It is interesting to notice that the frequency $\omega_{0}=1 / \tau$ increases as the moisture content increases. This implies that the effective permittivity tends towards $\epsilon_{\mathrm{s}}$ in the frequency band exploited for the measurement and, consequently, the parameters $\epsilon_{\infty}$ becomes difficult to determine, which is reflected by the uncertainties in Tab. 3 .

Table 3: Debye parameters for MCC with different moisture content

\begin{tabular}{llll} 
Set & $\epsilon_{\mathrm{s}}[-]$ & $\epsilon_{\infty}[-]$ & $\tau[\mathrm{ps}]$ \\
\hline A & $3.62 \pm 0.02$ & $2.63 \pm 0.03$ & $32.0 \pm 0.9$ \\
B & $4.27 \pm 0.04$ & $2.85 \pm 0.05$ & $24.2 \pm 0.8$ \\
C & $4.88 \pm 0.05$ & $2.79 \pm 0.12$ & $15.5 \pm 0.8$ \\
D & $5.49 \pm 0.07$ & $2.36 \pm 0.26$ & $10.3 \pm 0.8$ \\
\hline
\end{tabular}

It should be emphasized that the measurement is performed in the frequency band from $2.7 \mathrm{GHz}$ to $5.1 \mathrm{GHz}$ and, consequently, the permittivity outside this frequency band may deviate substantially from extrapolated values derived from the estimated parameters in Tab. 3. Gradinarsky et al. [3] used an open-ended coaxial probe for local in situ measurements of moisturized MCC pellets in the frequency band from $1 \mathrm{GHz}$ to $19 \mathrm{GHz}$. Their study indicates that the dispersion characteristics of MCC does not follow a Debye model for such a large frequency band and, therefore, it may be tempting to exploit more elaborate models such as the Havriliak-Negami response (18). Thus, we attempted to fit more elaborate the Cole-Cole and Cole-Davidson models to the measured data. The additional degree of freedom, i.e. $\alpha$ for the Cole-Cole model and $\beta$ for the Cole-Davidson model, reduces the residual and, unfortunately, it also introduces very large uncertainties in the values for $\epsilon_{\infty}$ and $\tau$, where the exponent $\beta$ is also very uncertain for the ColeDavidson model.

We conclude that for the estimation of moisture content of MCC with the measurement equipment (and frequency band 2.7-5.1 GHz) used in this article, it is sufficient to use a (basic) Debye model where both $\epsilon_{\mathrm{s}}$ and $\tau$ depend monotonically on the moisture content in a reliable manner. The optical response $\epsilon_{\infty}$ may be used for moisture contents below $15 \%(\mathrm{w} / \mathrm{w})$.

Next, we present the real and imaginary part of the effective permittivity as a function of frequency: (i) sample set $\mathrm{A}$ is shown in Fig. 11; (ii) sample set B is shown in Fig. 12; (ii) sample set $\mathrm{C}$ is shown in Fig. 13; and (iv) sample set D is shown in Fig. 14. In Figs. 11-14, the estimated permittivity is represented by four different parameterizations: (i) solid curve - piecewise constant permittivity; (ii) dashed curve - Cole-Cole model; (iii) dash-dotted curve - Cole-Davidson model; and (iv) dotted curve - Debye model. The piecewise-constant permittivity is described as $\epsilon_{\mathrm{c}}=\epsilon_{0}\left(\epsilon^{\prime}-j \epsilon^{\prime \prime}\right)$ with separate and independent values for $\epsilon^{\prime}$ and $\epsilon^{\prime \prime}$ in each frequency subinterval $f_{i}<f<f_{i+1}$. Here, $f_{i}=(i-1) \Delta f$ with $\Delta f=\left(f_{\mathrm{U}}-f_{\mathrm{L}}\right) / N$ and $i=1, \ldots, N$, where the number of subintervals is $N=16$. In each of the Figs. 11-14, the upper and lower piecewise-constant solid curves indicate the uncertainty interval around the estimated piecewise-constant values shown by the centered solid curve. (Only the simple constraints $\epsilon^{\prime} \geq 1$ and $\epsilon^{\prime \prime} \geq 0$ have been used for the estimation of the piecewise-constant permittivity.)

It is observed that the Debye, Cole-Cole and Cole-Davidson models reside mainly inside the region described by the the piecewise constant permittivity and its upper and lower uncertainties. Also, we notice that the uncertainty interval for the piecewise constant permittivity increases in absolute numbers as the moisture content increases.

\section{Discussion}

The measurement technique presented in this article is intended for the monitoring of the fluidized beds that are featured in process equipment used in pharmaceutical industry. As a first step towards this goal, we have demonstrated that it is feasible to measure the effective dielectric constant of moisturized MCC pellets. Moreover, the dielectric constant can be related to the moisture content, which is an underlying parameter of interest from the application view-point. For future work, our measurement system is equipped with six waveguide ports that may provide useful information on the spatial distribution of the dielectric material in the measurement region.

The final industrial application requires that the electromagnetic field problem is treated in 3D and such a formulation requires the usage of vector finite elements to model the electromagnetic field [14]. Thus, the finite element model presented in Sec. 2 and the corresponding sensitivities described in Sec. 3.1 can be used with minor modifications for the corresponding $3 \mathrm{D}$ situation.

For comparative purposes, we have implemented both a conventional node-based FEM for the scalar Helmholtz equation expressed in terms $E_{z}$ and a FEM with curl-conforming elements for the vector Helmholtz equation expressed in terms of $\boldsymbol{H}=\hat{\boldsymbol{x}} H_{x}+\hat{\boldsymbol{y}} H_{y}$. The conventional formulation for $E_{z}$ exploits higher-order elements and, consequently, it can achieve highly accurate scattering parameters and sensitivities. Our formulation for the magnetic field is implemented for linear elements, which makes it a second-order accurate method, and we conclude that it compares well with the higher-order formulation for $E_{z}$. In Sec. 4, we have exploited the higher-order nodebased formulation for $E_{z}$ and, in conclusion, we find that it is feasible to achieve similar performance with a $3 \mathrm{D}$ formulation based on vector elements although the computational cost and the programming efforts are substantially higher. 


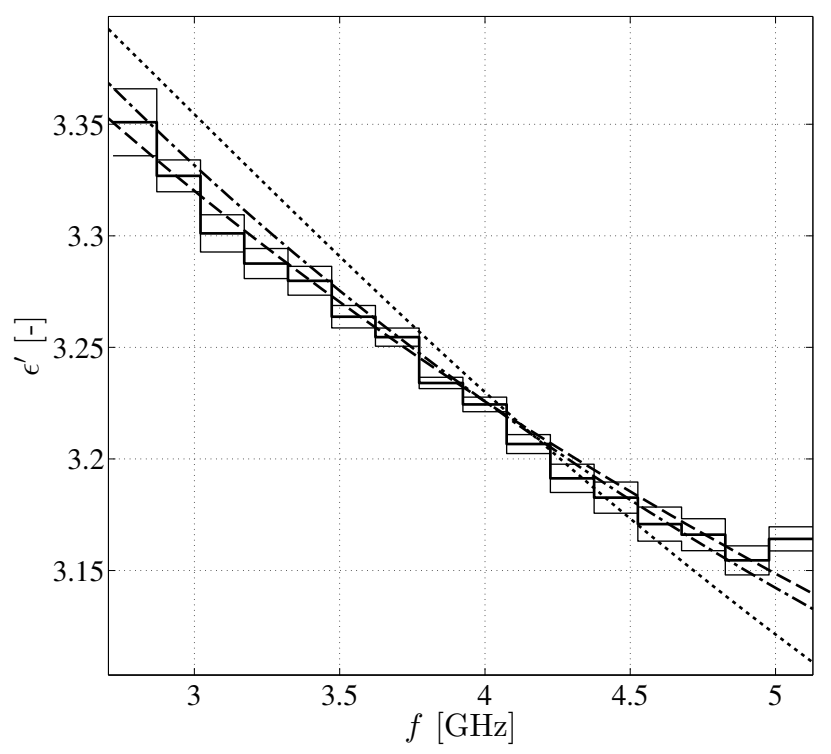

(a) Real part of the permittivity.

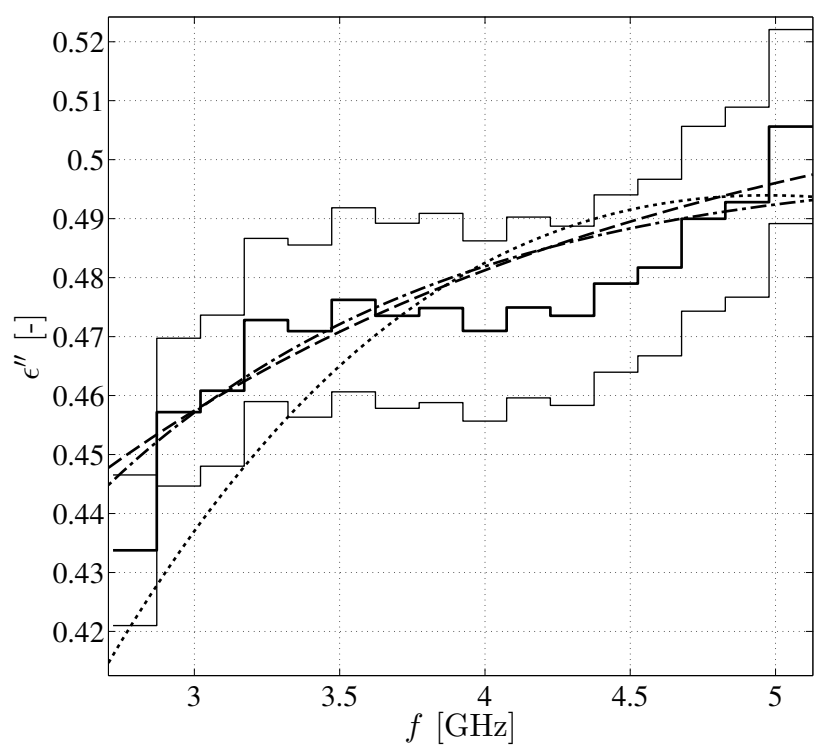

(b) Imaginary part of the permittivity.

Figure 11: MCC from sample set A: solid curve - piecewise constant permittivity; dashed curve - Cole-Cole model; dash-dotted curve - Cole-Davidson model; and dotted curve - Debye model.

\section{Conclusions}

We present a prototype microwave measurement system intended for the estimation of dielectric properties of moisturized microcrystalline cellulose (MCC) pellets, where we also demonstrate that the estimated dielectric properties depend on the moisture content of the MCC pellets. The measurement procedure is non-intrusive in the sense that the microwave sensors do not need to be in physical contact with the sample, which is a major advantage in most situations. Our measurement system operates in the frequency band from $2.7 \mathrm{GHz}$ to $5.1 \mathrm{GHz}$.

The measurement system is modeled by the finite element method in two-dimensions and the computational model compares well with experimental results for well-established test

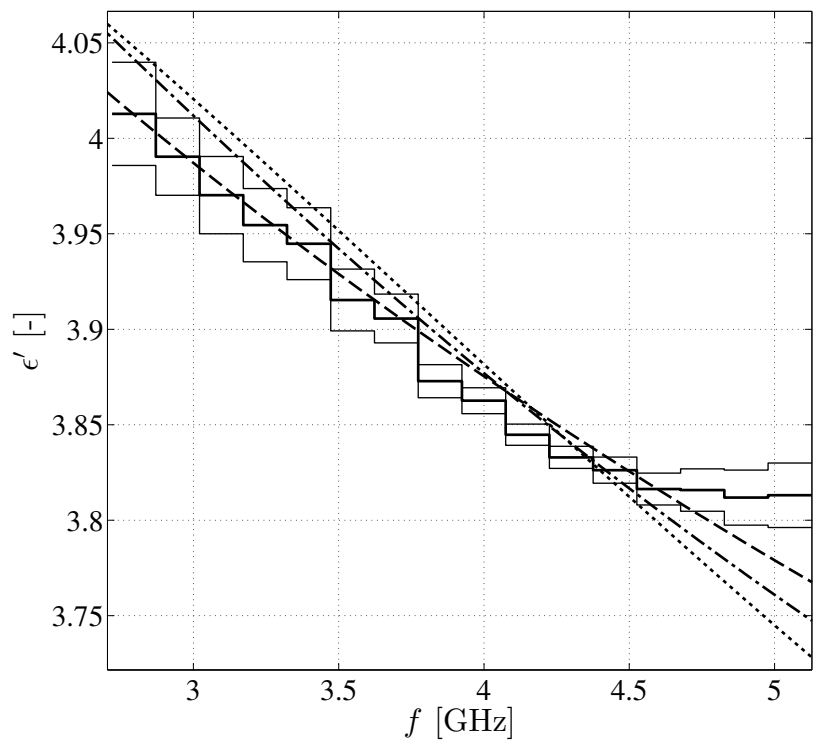

(a) Real part of the permittivity.

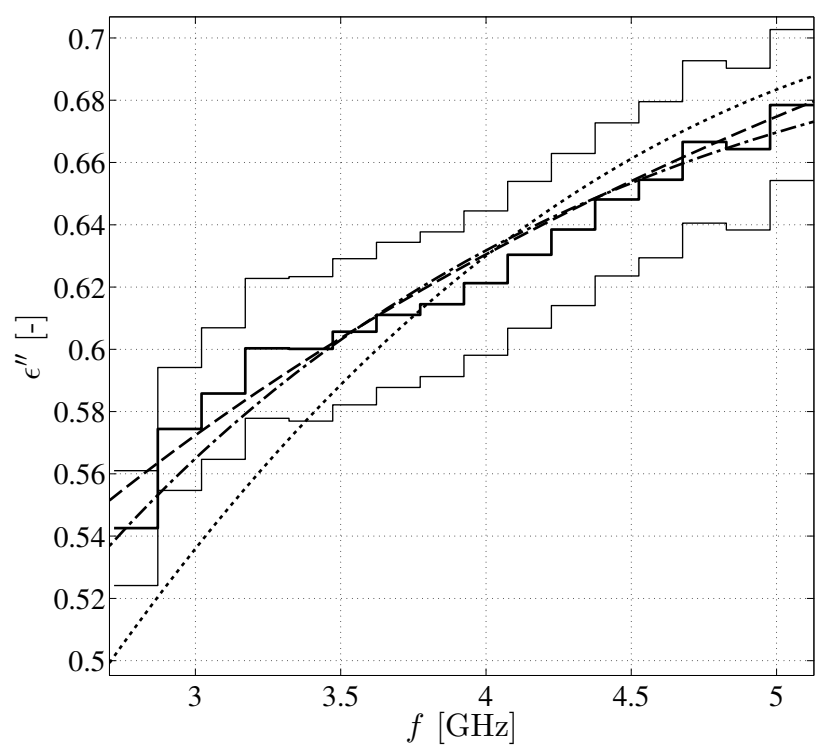

(b) Imaginary part of the permittivity.

Figure 12: MCC from sample set B: solid curve - piecewise constant permittivity; dashed curve - Cole-Cole model; dash-dotted curve - Cole-Davidson model; and dotted curve - Debye model.

cases. The permittivity is estimated by means of minimizing the deviation between (i) the measured scattering parameters and (ii) the computed scattering parameters, where the computational model features the unknown permittivity as an input parameter. We use gradient-based optimization to determine the unknown permittivity, where the sensitivities are formulated in terms of the original field solution and an adjoint field solution. The sensitivities are also used to estimate uncertainties in the permittivity based on uncertainties in, e.g., the size of the dielectric sample under test.

We estimate the effective complex permittivity described by a Debye model for densely packed MCC pellets that feature different levels of moisture content with a few samples in the 


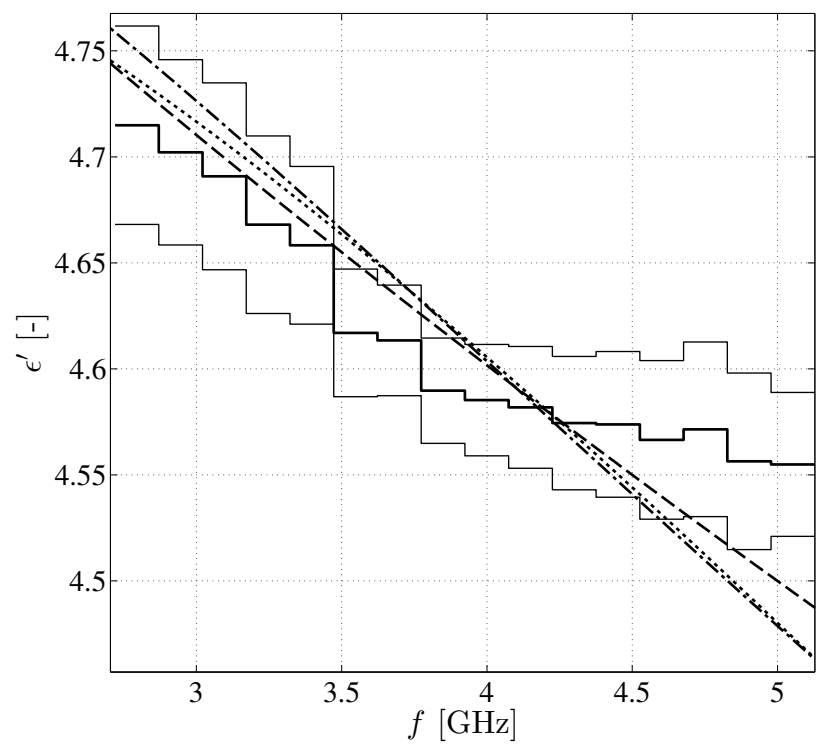

(a) Real part of the permittivity.

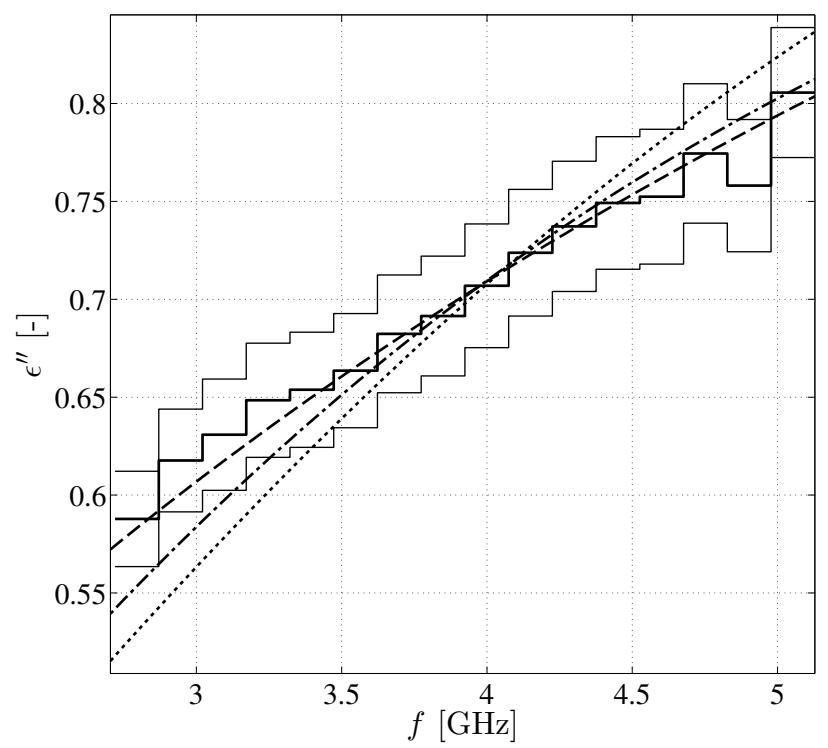

(b) Imaginary part of the permittivity.

Figure 13: MCC from sample set C: solid curve - piecewise constant permittivity; dashed curve - Cole-Cole model; dash-dotted curve - Cole-Davidson model; and dotted curve - Debye model.

interval from $9 \%$ to $23 \%(\mathrm{w} / \mathrm{w})$. It is found that the parameters in the Debye model can be estimated to an accuracy of $3 \%$ for the lowest moisture content and that the measurement uncertainties increase with moisture content such that the results for the highest moisture content yields an accuracy of about $13 \%$. It is found that the static permittivity and the relaxation time associated with the Debye model vary monotonically with moisture content, which makes it feasible to use these parameters for the estimation of moisture content of the MCC.

\section{Acknowledgments}

Livia Cerullo was financially supported by AstraZeneca. In addition, Johan Winges was financially supported by the

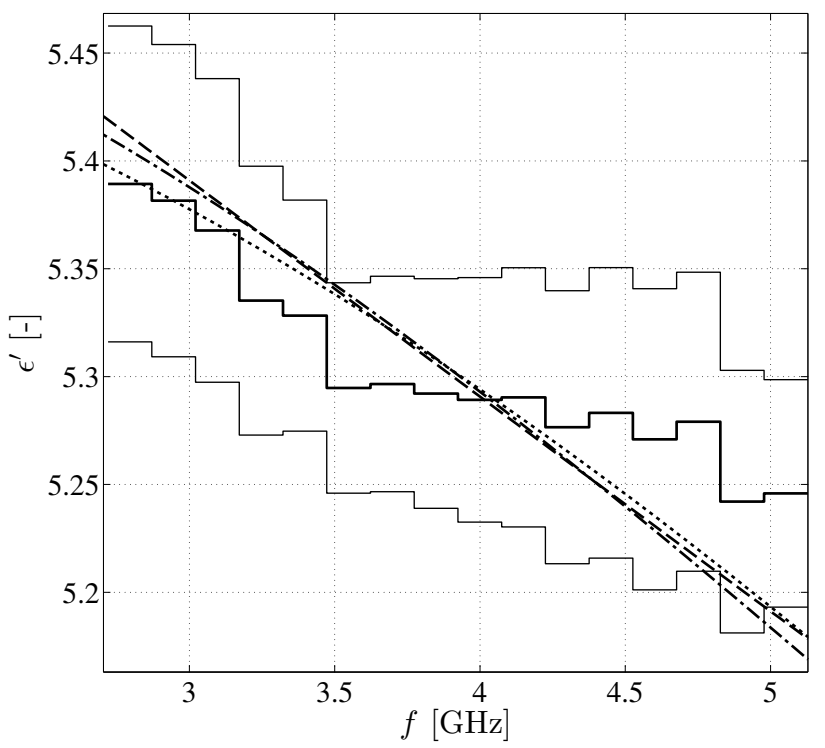

(a) Real part of the permittivity.

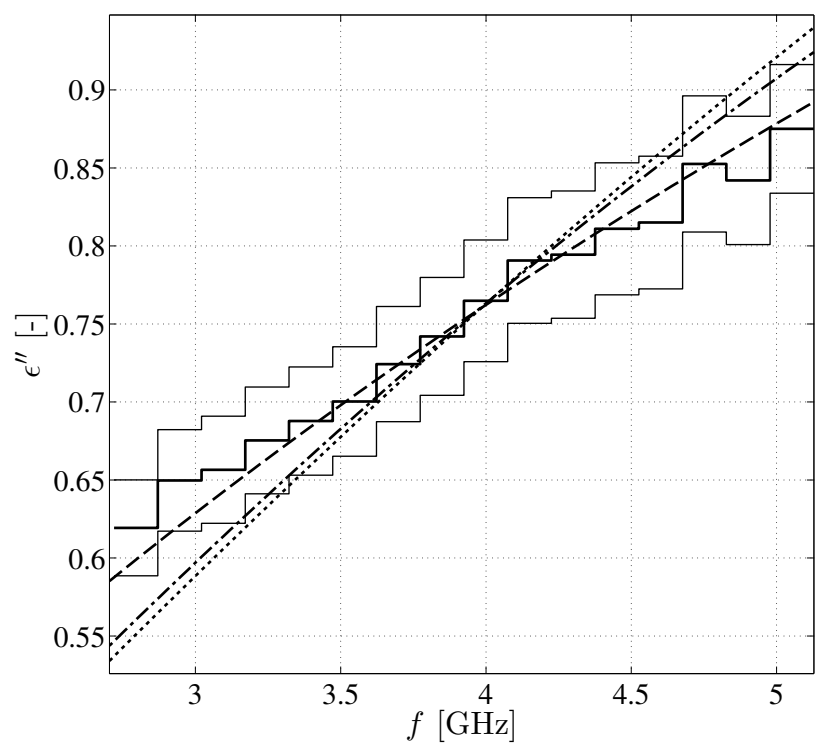

(b) Imaginary part of the permittivity.

Figure 14: MCC from sample set D: solid curve - piecewise constant permittivity; dashed curve - Cole-Cole model; dash-dotted curve - Cole-Davidson model; and dotted curve - Debye model.

Swedish Research Council (dnr 2010-4627) in the project "Model-based Reconstruction and Classification Based on Near-Field Microwave Measurements". The computations were performed on resources at Chalmers Centre for Computational Science and Engineering (C3SE) provided by the Swedish National Infrastructure for Computing (SNIC).

\section{References}

[1] C. A. M. da Silva, J. J. Butzge, M. Nitz, O. P. Taranto, Monitoring and control of coating and granulation processes in fluidized beds - a review, Adv. Powder Technol. 25 (2014) 195-210. doi:10.1016/j.apt.2013.04.008.

[2] G. Smith, Dielectric analysis of water in microcrystalline cellulose, 
Pharm. Pharmacol. Commun. 1 (9) (1995) 419-422. doi:10.1111/j.20427158.1995.tb00455.x.

[3] L. Gradinarsky, H. Brage, B. Lagerholm, I. N. Björn, S. Folestad, In situ monitoring and control of moisture content in pharmaceutical powder processes using an open-ended coaxial probe, Meas. Sci. Technol. 17 (2006) 1847-1853. doi:10.1088/0957-0233/17/7/024.

[4] L. Obregòn, L. Quiñones, C. Velázquez, Model predictive control of a fluidized bed dryer with an inline NIR as moisture sensor, Control Eng Pract 21 (2013) 509-517. doi:10.1016/j.conengprac.2012.11.002.

[5] J. Rantanen, S. Lehtola, P. Rämet, J.-P. Mannermaa, J. Yliruusi, On-line monitoring of moisture content in an instrumented fluidized bed granulator with a multi-channel NIR moisture sensor, Powder Technol. 99 (1998) 163-170. doi:10.1016/s0032-5910(98)00100-4.

[6] P. Frake, D. Greenhalgh, S. M. Grierson, J. M. Hempenstall, D. R. Rudd, Process control and end-point determination of a fluid bed granulation by application of near infra-red spectroscopy, Int. J. Pharm. 151 (1997) 75-80. doi:10.1016/s0378-5173(97)04894-1.

[7] T. Dyakowski, L. F. C. Jeanmeure, A. J. Jaworski, Applications of electrical tomography for gas-solids and liquid-solids flows - a review, Powder Technol. 112 (2000) 174-192. doi:10.1016/s0032-5910(00)00292-8.

[8] H. G. Wang, P. R. Senior, R. Mann, W. Q. Yang, Online measurements and control of solids moisture in fluidised bed dryers, Chem. Eng. Sci. 64 (2009) 2893-2902. doi:10.1016/j.ces.2009.03.014.

[9] H. Wang, L. Gradinarsky, S. Folestad, L. Cerullo, Imaging pharmaceutical multi-processes in fluidized bed by electrical capacitance tomography, in: AIChE Annual Meeting, USA, 2010.

[10] V. Rimpiläinen, L. M. Heikkinen, M. Vauhkonen, Moisture distribution and hydrodynamics of wet granules during fluidized-bed drying characterized with volumetric electrical capacitance tomography, Chem. Eng. Sci. 75 (2012) 220-234. doi:10.1016/j.ces.2012.03.028.

[11] C. Buschmüller, W. Wiedey, C. Döscher, J. Dressler, J. Breitkreutz, Inline monitoring of granule moisture in fluidized-bed dryers using microwave resonance technology, Eur. J. Pharm. Biopharm. 69 (2008) 380387. doi:10.1016/j.ejpb.2007.09.014.

[12] L. Cerullo, J. Nohlert, J. Winges, T. Rylander, T. McKelvey, L. Gradinarsky, M. Viberg, S. Folestad, Microwave measurements for metal vessels, in: Proc. of the 7th Europ. Conf. on Antennas and Propagation EuCAP, 2013, pp. 3869-3873.

[13] J. Nohlert, L. Cerullo, J. Winges, T. Rylander, T. McKelvey, A. Holmgren, L. Gradinarsky, S. Folestad, M. Viberg, A. Rasmuson, Global monitoring of fluidized-bed processes by means of microwave cavity resonances, Measurement 55 (2014) 520-535. doi:10.1016/j.measurement.2014.05.034.

[14] J. M. Jin, The Finite Element Method in Electromagnetics (2nd Edition), New York, NY: John Wiley \& Sons, 2002.

[15] D. M. Pozar, Microwave engineering, New York, NY: Wiley, 1998.

[16] F. Alimenti, P. Mezzanotte, L. Roselli, R. Sorrentino, A revised formulation of model absorbing and matched modal source boundary conditions for the efficient FDTD analysis of waveguide structures, IEEE Trans. Microw. Theory Techn. 48 (1) (2000) 50-59.

[17] K. Holmström, A. O. Göran, M. M. Edvall, User's Guide for TOMLAB 7, Tomlab Optimization AB, Västerås Technology Park, Trefasgatan 4, SE72130 Västerås, Sweden (2009).

[18] L. Cerullo, T. Rylander, M. Viberg, Determination of model order for inverse scattering applications, in: Proc. of the V European Conf. on Computational Fluid Dynamics ECCOMAS CFD 2010, 2010, p. 01314.

[19] M. Gustafsson, S. He, An optimization approach to two-dimensional time domain electromagnetic inverse problems, Radio Science 35 (2) (2000) 525-536. doi:10.1029/1999rs900091.

[20] E. Wadbro, M. Berggren, High contrast microwave tomography using topology optimization techniques, J. Comput. Appl. Math. 234 (2010) 1773-1780. doi:10.1016/j.cam.2009.08.027.

[21] L. Garnero, A. Franchois, J.-P. Hugonin, C. Pichot, N. Joachimowicz, Microwave imaging - complex permittivity reconstruction by simulated annealing, IEEE Trans. Microw. Theory Techn. 39 (1991) 1801-1807. doi:10.1109/22.97480.

[22] S. Havriliak, S. Negami, A complex plane representation of dielectric and mechanical relaxation processes in some polymers, Polymer 8 (1967) $161-210$.

[23] A. Sihvola, Electromagnetic Mixing Formulas and Applications, Herts: IET, 2008.
[24] A. Bondeson, Y. Yang, P. Weinerfelt, Shape optimization for radar cross sections by a gradient method, Int. J. Numer. Meth. Engng. 61 (5) (2004) 687-715. doi:10.1002/nme.1088.

[25] E. Nyfors, P. Vainikainen, Industrial microwave sensors, Norwood, MA: Artech House, 1989.

[26] R. Afzalzadeh, Dielectric constant measurements of finite-size sheet at microwave frequencies by pseudo-brewster's angle method, IEEE Trans. Microw. Theory Techn. 46 (9) (1998) 1307-1309. doi: $10.1109 / 22.709475$.

[27] K. Dudeck, L. Buckley, Dielectric material measurement of thin samples at millimeter wavelengths, IEEE Trans. Instrum. Meas. 41 (5) (1992) 723-725. doi:10.1109/19.177352. 